\title{
Association between Polymorphisms of Antioxidant Gene (MnSOD, CAT, and GPx1) and Risk of Coronary Artery Disease
}

\author{
Hseng-Long Yeh, ${ }^{1,2}$ Li-Tang Kuo, ${ }^{3,4}$ Fung-Chang Sung, ${ }^{5,6}$ and Chih-Ching Yeh $\mathbb{D}^{1,7}$ \\ ${ }^{1}$ School of Public Health, College of Public Health, Taipei Medical University, Taipei, Taiwan \\ ${ }^{2}$ Division of Cardiology, Department of Internal Medicine, Sijhih Cathay General Hospital, Sijhih City, Taipei, Taiwan \\ ${ }^{3}$ Division of Cardiology, Department of Internal Medicine, Chang Gung Memorial Hospital, Keelung, Taiwan \\ ${ }^{4}$ Chang Gung University College of Medicine, Taoyuan, Taiwan \\ ${ }^{5}$ Management Office for Health Data, China Medical University Hospital, Taichung, Taiwan \\ ${ }^{6}$ Graduate Institute of Clinical Medical Science, School of Medicine, College of Medicine, China Medical University, Taichung, Taiwan \\ ${ }^{7}$ Department of Public Health, China Medical University, Taichung, Taiwan \\ Correspondence should be addressed to Chih-Ching Yeh; ccyeh@tmu.edu.tw
}

Received 11 May 2018; Revised 25 July 2018; Accepted 2 August 2018; Published 26 August 2018

Academic Editor: Achuthan C. Raghavamenon

Copyright (C) 2018 Hseng-Long Yeh et al. This is an open access article distributed under the Creative Commons Attribution License, which permits unrestricted use, distribution, and reproduction in any medium, provided the original work is properly cited.

\begin{abstract}
Objective. Reactive oxygen species (ROS) been cited as one of the major causes of atherosclerosis and coronary artery disease which are possible agents inducing DNA damage. Manganese superoxide dismutase (MnSOD), catalase (CAT), and glutathione peroxidase-1 (GPxl) have evolved to address primary defense against free radical mediated damage in mitochondria. The aim of this study was to delineate the association of MnSOD, CAT, and GPx1 polymorphisms and risk of CAD in Taiwan. Methods. We conducted a case-control study with 657 participants recruited at a medical center. All subjects were evaluated by noninvasive stress test and then quantitative coronary angiography to confirm the diagnosis of CAD. 447 CAD cases were defined as $>50 \%$ stenosis of coronary artery and 210 controls were stenosed below 50\%. Polymorphisms of MnSOD (Val16Ala), CAT (C-262T), and GPxl (Pro198Leu) genes were determined by polymerase chain reaction methods. Multivariate logistic regression model was used to calculate the odds ratios (ORs) and 95\% confidence intervals (CIs). Results. The MnSOD Val/Ala+Ala/Ala genotype was significantly associated with an increased risk of CAD compared to the Val/Val genotype $(\mathrm{OR}=1.86,95 \% \mathrm{CI}=1.15-3.01)$. This polymorphism was also associated with the severity of CAD of single and two vessel diseases. The corresponding ORs were 2.31 ( $95 \% \mathrm{CI}=1.32-4.03)$ and $1.92(95 \% \mathrm{CI}=1.02-3.61)$, respectively. Among cigarette smokers, the harmful genetic effect of $M n S O D$ Ala allele on CAD risk was much higher $(\mathrm{OR}=2.23,95 \% \mathrm{CI}=1.02-4.88)$. However, the interaction between $M n S O D$ genotype and cigarette smoking on CAD risk was not significant. No significant association between CAT and GPxl polymorphisms and CAD risk was observed. Conclusion. Our results suggest that $M n S O D$ polymorphism is an independent risk factor for susceptibility to $\mathrm{CAD}$ in the Chinese population.
\end{abstract}

\section{Introduction}

Cardiovascular disease (CVD) is the leading cause of mortality worldwide. Based on the World Health Organization (WHO) data in 2013, an estimated one-third of global deaths were attributable to CVDs $[1,2]$. Through large-scale epidemiologic studies, several conventional risk factors for CVDs have been identified for instance, cigarette smoking, hypertension, hyperlipidemia, diabetes mellitus, and obesity [3]. An intensified intervention strategy, treating multiple risk factors to target, brings about a 20 percent reduction in cardiovascular events in high risk individuals [4].

However, some discovered that these well-known risk factors merely account for $50-80 \%$ of CVDs $[5,6]$ and still around $15-20 \%$ of myocardial infarction occurred in individuals lacking any of the traditional risks [5]. Further studies to investigate novel CVDs risk markers and thereby early recognizing preclinical disease are essential.

Since the initial human genome sequencing in 2001 [7] and publication of HapMap in 2005 [8], the development in 
genetic analysis and personalized precision medicine have rapidly progressed. Efforts on genetic basis for CVDs have been made, including genome-wide association study [9], but suffered from inconsistent results and still have a long way to go to make a conclusion on how genetic factors affect CVDs. Our study group had previously recruited a total of $458 \mathrm{CAD}$ patients and 209 health subjects and found a null association between glutathione-S-transferase genes and susceptibility to CAD [10].

Since mitochondria make up a large mass, approximately $30 \%$ of the myocyte, oxidative stress to mitochondria plays important roles in pathophysiology of CVDs [11]. Oxidative stress might come from overproduction of ROS or inadequate antioxidant functions. MnSOD, CAT, and GPxl are mitochondrial enzymes, which act on the ROS metabolism and participate in the cellular defence to oxidative stress [12]. The primary antioxidant in the mitochondria that is MnSOD and CAT converts ROS into oxygen and hydrogen peroxide. The second line of enzymatic antioxidant defense is GPxl isoenzymes. To make further investigation on genetic predispositions of CAD, we have conducted a research on antioxidant genes polymorphism and CAD risk. The aim of the study is to investigate the association of SNPs such as $M n S O D, C A T$, and GPX1 with risk of CAD among a population from Taiwan.

\section{Materials and Methods}

2.1. Subjects. Detailed descriptions of the specific characteristics of the study participants have been published previously [13]. In brief, participants were recruited from the Chang Gung Memorial Hospital Taiwan. The CAD participants in this study were angiographically confirmed by experienced cardiologists. Individuals with at least one coronary artery diameter stenosis $\geq 50 \%$ were defined as cases $(n=481)$ and the others as control group $(n=228)$ [14]. This study protocol had been proven by The Institutional Review Board at the Chang Gung Memorial Hospital and all patients provided informed consents.

Before receiving the angiography, each participant completed a self-administered questionnaire covering sociodemographic characteristics, tobacco and alcohol use, height, weight, family CAD history, and personal medical history of hypertension and diabetes. Individuals with blood pressure $\geq 140 / 90 \mathrm{mmHg}$ or receiving antihypertensive therapy were considered as hypertensive. Those with fasting plasma glucose $>126 \mathrm{mg} / \mathrm{dl}$ or receiving glucose lowering treatment were considered as diabetes. Body mass index (BMI) was calculated as weight/height ${ }^{2}\left(\mathrm{~kg} / \mathrm{m}^{2}\right)$. Cigarette smoking and alcohol drinking were classified into three groups: current users, nonusers, and ex-users (who had stopped use for at least six months). Blood samples of overnight fast (12 to 14 hours) provided by subjects were measured for the biochemical profiles by the Clinical Chemistry Department at the hospital, including total cholesterol, high-density lipoprotein cholesterol (HDL-C), uric acid, creatinine, and triglyceride using standard enzymatic methods [15]. The low-density lipoprotein cholesterol (LDL-C) levels were calculated from the Friedewald formula. Non-HDL-C was equal to the level of cholesterol minus HDL-C. Triglyceride-rich lipoprotein cholesterol (TRL-C) was calculated by total cholesterol minus levels of LDL-C and HDL-C.

2.2. Genotyping. Genomic DNA was extracted from buffy coat cells using a DNA extraction kit (Qiagen, Inc., Chatsworth CA). The MnSOD Val-9Ala (rs4880), CAT C262T (rs1001179), and GPx1 Pro198Leu (rs1050450) polymorphisms were determined using polymerase chain reaction (PCR) and restriction fragment length polymorphism (RFLP) methods [16, 17]. All PCR reactions were performed in a $20 \mu \mathrm{l}$ final volume containing $0.25 \mu \mathrm{M}$ of each primer, 50 ng genomic DNA, $1.5 \mathrm{mM} \mathrm{MgCl}_{2}, 0.2 \mathrm{mM}$ dNTPs, and 1.0 unit of Taq DNA Polymerase in the buffer provided by the manufacturer. Amplification was performed in a Mastercycler gradient thermocycler (Eppendorf, Hamburg, Germany) for the PCR reaction.

The 112 bp MnSOD Val-9Ala PCR products were amplified with the primers $5^{\prime}$-GCA CCA GCA GGC AGC TGG CGC CGG-3 ${ }^{\prime}$ (sense) and $5^{\prime}$-TGC GCG TTG ATG TGA GGT TCC AG-3' (antisense) and digested with NgoMIV (New England BioLabs, Beverly, MA, USA). The Ala allele revealed 90 and $22 \mathrm{bp}$ fragments following digestion and polyacrylamide gel electrophoresis, whilst the Val allele was not digested by NgoMIV [18]. The 183 bp CAT C-262T PCR products were amplified with the primers $5^{\prime}$-TAA GAG CTG AGA AAG CAT AGC T-3' (sense) and $5^{\prime}$-AGA GCC TCG CCC CGC CGG ACC G-3' (antisense) and digested with SmaI (Fermentas, Glen Burnie, MD, USA). The C allele revealed 153 and $30 \mathrm{bp}$ fragments following digestion and polyacrylamide gel electrophoresis, while the $\mathrm{T}$ allele was not digested by SmaI [19]. The 337 bp GPx1 Pro198Leu PCR products were amplified with the primers $5^{\prime}$-TGT GCC CCT ACG GTA CA-3' (sense) and $5^{\prime}$-CCA AAT GAC AAT GAC ACA GG $-3^{\prime}$ (antisense) and digested with ApaI (Fermentas, USA). The Pro allele revealed 258 and 79 bp fragments following digestion and polyacrylamide gel electrophoresis, whilst the Lys allele was not able to be digested by ApaI. For quality control, $10 \%$ replicate samples were included with the study samples and showed $100 \%$ concordance for all polymorphisms. As well, all laboratory personnel were blinded to the CAD status of the participants.

2.3. Statistical Analysis. A total of 52 subjects were excluded because of genotyping failure ( 34 cases, 18 controls), leaving 447 cases and 210 controls for the final analysis. Tests for Hardy-Weinberg equilibrium amongst controls were conducted using Chi-square test featuring one degree of freedom. Differences between cases and controls were compared by either Student's $t$-test or Wilcoxon rank-sum test for continuous variables or Chi-square test for qualitative data. Unconditional logistic regression was used to calculate the odd ratios (ORs) and 95\% confidence intervals (CIs) for the CAD risk associated with the MnSOD, CAT, and GPxl genotypes. The adjusted $\mathrm{OR}$ of genotypes was calculated using a multivariate logistic regression model adjusted for age, sex, cigarette smoking (never and ever), and alcohol drinking (never and ever). Interaction analysis based on a multiplicative scale was conducted to evaluate the gene-environment interaction 
TABLE 1: Demographic and clinical characteristics of the study population.

\begin{tabular}{|c|c|c|c|}
\hline Variables $^{\mathrm{a}}$ & Cases, $\mathrm{n}(\%) \mathrm{N}=447$ & Controls, $\mathrm{n}(\%) \mathrm{N}=210$ & p-value ${ }^{b}$ \\
\hline Male & $329(73.6)$ & $130(61.9)$ & 0.002 \\
\hline Age (mean $\pm S D)$, years & $64.8 \pm 12.2$ & $58.1 \pm 12.6$ & $<0.001$ \\
\hline $\mathrm{BMI}($ mean $\pm \mathrm{SD}), \mathrm{kg} / \mathrm{m}^{2}$ & $25.3 \pm 4.03$ & $25.5 \pm 3.8$ & 0.546 \\
\hline Stenosis $1 / 2$ / 3 & $159 / 129 / 159$ & & \\
\hline Ethnic group & & & 0.591 \\
\hline Taiwanese & $336(76.9)$ & $165(79.7)$ & \\
\hline Mainland Chinese & $59(13.5)$ & $27(13.0)$ & \\
\hline Other $^{c}$ & $42(9.6)$ & $15(7.3)$ & \\
\hline Occupation & & & 0.390 \\
\hline White-collar & 73(17.1) & $27(13.0)$ & \\
\hline Blue-collar & $141(32.9)$ & $69(33.2)$ & \\
\hline Other $^{\mathrm{d}}$ & $214(50.0)$ & $112(53.8)$ & \\
\hline Family CAD history & $104(24.9)$ & $44(23.0)$ & 0.508 \\
\hline History of hypertension & $229(51.2)$ & $84(40.0)$ & 0.007 \\
\hline History of DM & $173(38.7)$ & $24(11.4)$ & $<0.001$ \\
\hline Cigarette smoking & & & $<0.001$ \\
\hline None & $194(43.5)$ & $129(61.4)$ & \\
\hline Current smokers & $195(43.7)$ & $59(28.1)$ & \\
\hline Ex-Smokers & $57(12.8)$ & $22(10.5)$ & \\
\hline Alcohol drinking & & & 0.853 \\
\hline None & $344(77.5)$ & $157(75.8)$ & \\
\hline Current drinkers & $97(21.8)$ & $48(23.2)$ & \\
\hline Ex-drinkers & $3(0.7)$ & $2(1.0)$ & \\
\hline Creatinine (mean $\pm \mathrm{SD}), \mathrm{mg} / \mathrm{dl}$ & $2.0 \pm 2.6$ & $1.4 \pm 2.0$ & 0.005 \\
\hline Uric acid (mean $\pm \mathrm{SD}), \mathrm{mg} / \mathrm{dl}$ & $8.0 \pm 2.5$ & $7.3 \pm 2.1$ & $<0.001$ \\
\hline HDL-C (mean \pm SD), mg/dl & $34.7 \pm 12.2$ & $37.6 \pm 15.0$ & 0.009 \\
\hline $\mathrm{LDL}-\mathrm{C}($ mean $\pm \mathrm{SD}), \mathrm{mg} / \mathrm{dl}$ & $175.4 \pm 54.0$ & $157.9 \pm 41.7$ & $<0.001$ \\
\hline Cholesterol (mean \pm SD), mg/dl & $244.2 \pm 61.2$ & $219.2 \pm 45.2$ & $<0.001$ \\
\hline Non-HDL-C (mean \pm SD), mg/dl & $211.8 \pm 59.3$ & $185.9 \pm 43.1$ & $<0.001$ \\
\hline TRL-C (mean \pm SD), mg/dl & $35.4 \pm 38.3$ & $27.5 \pm 28.8$ & 0.006 \\
\hline Triglyceride [median (IQR)], mg/dl & $153(100-239)$ & $112.0(76-184)$ & $<0.001$ \\
\hline
\end{tabular}

TRL-C, triglyceride-rich lipoprotein cholesterol; IQR, interquartile range.

a: numbers not equal to total number were due to missing data.

b: Student's $t$-test, Wilcoxon rank-sum test or Chi-squared test.

c: including Hakka and aborigines.

$\mathrm{d}$ : including retiree and housekeeper.

for the CAD risk. All analyses were performed using the Statistical Analysis System version 9.1.3 (SAS Institute, Cary, $\mathrm{NC}$ ) and all tests were 2-sided with $\mathrm{P}$-value $<0.05$ as the significant level.

\section{Results}

The demographic and clinical characteristics of the participants in the study are summarized in Table 1. Compared with the controls, the CAD cases were primarily male, older, smokers, and presenting hypertension and diabetes $(\mathrm{p}<$ 0.05). The study cases also had higher levels of creatinine, uric acid, LDL-C, cholesterol, non-HDL-C, TRL-C, and triglyceride, but lowered HDL-C levels.

The genotypic distributions of the MnSOD, CAT, and GPxl polymorphisms for both cases and controls are shown in Table 2. The allele frequencies for the $M n S O D$ Ala allele, CAT $\mathrm{T}$ allele, and GPX1 Leu allele amongst the controls were 7.38, 3.1, and $4.52 \%$, respectively. All these polymorphisms are in Hardy-Weinberg equilibrium. Because of the low frequencies of variant alleles for these three polymorphisms, we divided each polymorphism into wild and variant genotype for further data analyses (i.e., Val/Ala+Ala/Ala and Val/Val for MnSOD, C/T+T/T and T/T for CAT, and Pro/Leu and Pro/Pro for GPx1). After controlling for covariates, the CAD risk was statistically significantly increased by $\mathrm{MnSOD} \mathrm{Val} / \mathrm{Ala}+\mathrm{Ala} / \mathrm{Ala}$ genotype $(\mathrm{OR}=1.86,95 \% \mathrm{CI}=1.15$ 3.01), compared to $M n S O D \mathrm{Val} / \mathrm{Val}$ genotype. A moderately increased risk for $\mathrm{CAD}$ was also observed in subjects with CAT $\mathrm{C} / \mathrm{T}+/ \mathrm{T} / \mathrm{T}$ genotype than those with $\mathrm{C} / \mathrm{C}$ genotype (OR $=1.51,95 \% \mathrm{CI}=0.77-2.95)$. However, the GPxl polymorphism was not associated with CAD risk. 
TABLE 2: Association between MnSOD, CAT, and GPxl genotypes and risk of coronary artery disease.

\begin{tabular}{lccc}
\hline Genotype & Cases, $\mathrm{n}(\%) \mathrm{N}=447$ & Controls, $\mathrm{n}(\%) \mathrm{N}=210$ & $\mathrm{OR}_{(95 \% \mathrm{CI})^{\mathrm{a}}}$ \\
\hline MnSOD Val-9Ala & & & 1.00 \\
Val/Val & $352(78.7)$ & $181(86.2)$ & $1.82(1.10-2.99)$ \\
Val/Ala & $83(18.6)$ & $27(12.9)$ & $2.40(0.51-11.3)$ \\
Ala/Ala & $12(2.7)$ & $2(0.9)$ & 0.012 \\
P for trend & & & 1.00 \\
Val/Val & $352(78.8)$ & $181(86.2)$ & 0.019 \\
Val/Ala+Ala/Ala & $95(21.2)$ & $29(13.8)$ & $1.86(1.15-3.01)$ \\
CAT C-262T & & & 1.00 \\
C/C & $403(90.2)$ & $197(93.8)$ & $1.43(0.73-2.82)$ \\
C/T & $42(9.4)$ & $13(6.2)$ & - \\
T/T & $2(0.5)$ & $0(0.0)$ & 1.00 \\
C/C & $403(90.2)$ & $197(93.8)$ & $1.51(0.77-2.95)$ \\
C/T+T/T & $44(9.8)$ & $13(6.2)$ & 0.299 \\
GPxl Pro198Leu & & & 0.982 \\
Pro/Pro & $406(90.8)$ & $191(90.9)$ & 1.00 \\
Pro/Leu & $41(9.2)$ & $19(9.1)$ & $1.08(0.58-1.99)$ \\
\hline
\end{tabular}

a: adjusted for age, sex, cigarette smoking, and alcohol drinking.

TABLE 3: Association between MnSOD, CAT, and GPxl genotypes and risk of coronary artery disease severity.

\begin{tabular}{|c|c|c|c|c|}
\hline \multirow{2}{*}{ Genotype } & \multicolumn{4}{|c|}{ CAD severity } \\
\hline & 0 & 1 & 2 & 3 \\
\hline \multicolumn{5}{|l|}{ MnSOD } \\
\hline $\mathrm{Val} / \mathrm{Val}$ & 181 & 119 & 102 & 131 \\
\hline $\mathrm{Val} / \mathrm{Ala}+\mathrm{Ala} / \mathrm{Ala}$ & 29 & 40 & 27 & 28 \\
\hline OR $(95 \% C I)^{\mathrm{a}}$ & 1.00 & $2.31(1.32-4.03)$ & $1.92(1.02-3.61)$ & $1.40(0.75-2.61)$ \\
\hline $\mathrm{P}$-value & & 0.003 & 0.043 & 0.288 \\
\hline \multicolumn{5}{|l|}{ CAT } \\
\hline $\mathrm{C} / \mathrm{C}$ & 197 & 145 & 117 & 141 \\
\hline $\mathrm{C} / \mathrm{T}+\mathrm{T} / \mathrm{T}$ & 13 & 14 & 12 & 18 \\
\hline $\mathrm{OR}(95 \% \mathrm{CI})^{\mathrm{a}}$ & 1.00 & $1.25(0.55-2.83)$ & $1.40(0.58-3.34)$ & $1.80(0.82-3.99)$ \\
\hline $\mathrm{P}$-value & & 0.592 & 0.454 & 0.146 \\
\hline \multicolumn{5}{|l|}{ GPxl } \\
\hline Pro/Pro & 191 & 142 & 120 & 144 \\
\hline Pro/Leu & 19 & 17 & 9 & 15 \\
\hline $\mathrm{OR}(95 \% \mathrm{CI})^{\mathrm{a}}$ & 1.00 & $1.24(0.60-2.59)$ & $0.83(0.34-2.03)$ & $1.05(0.49-2.28)$ \\
\hline P-value & & 0.562 & 0.689 & 0.895 \\
\hline
\end{tabular}

a: adjusted for age, sex, cigarette smoking, and alcohol drinking.

We also categorized all study subjects into four groups according to their CAD severity. As shown in Table 3, CAD risk is most profoundly increased with $M n S O D$ $\mathrm{Val} / \mathrm{Ala}+\mathrm{Ala} / \mathrm{Ala}$ polymorphisms in the one-vessel disease group $(\mathrm{OR}=2.31,95 \% \mathrm{CI}=1.32-4.03)$. Subjects with $M n S O D$ $\mathrm{Val} / \mathrm{Ala}+\mathrm{Ala} / \mathrm{Ala}$ polymorphisms also showed higher CAD risk, although less significant, in those with two-vessel disease $(\mathrm{OR}=1.92,95 \% \mathrm{CI}=1.02-3.06)$. There is no difference in CAD risk between wild and variant $M n S O D$ genotypes among the three-vessel disease group ( $\mathrm{OR}=1.40,95 \% \mathrm{CI}=0.75-2.61)$.

The interactions between $M n S O D, C A T$, and GPx1 polymorphisms and cigarette smoking for CAD risk are shown in
Table 4 . The increased CAD risk by $M n S O D$ Val/Ala+Ala/Ala genotype was pronounced among ever smokers, but this interaction was not significant ( $\mathrm{p}$ for interaction $=0.552$ ). Among the ever smokers, subjects carrying the MnSOD $\mathrm{Val} / \mathrm{Ala}+\mathrm{Ala} / \mathrm{Ala}$ genotype had 2.23-fold risk for CAD than those carrying the $\mathrm{Val} / \mathrm{Val}$ genotype $(\mathrm{OR}=2.23,95 \% \mathrm{CI}$ $=1.02-4.88$ ). However, there was no significant interaction between CAT and GPX1 polymorphisms and cigarette smoking

The smoking-stratified analysis for association between $\mathrm{MnSOD}$ genotypes and CAD severity was shown in Table 5. We found that variant $M n S O D$ genotypes are associated 
TABLE 4: Association between MnSOD, CAT, and GPx1 genotypes and risk of coronary artery disease stratified by cigarette smoking status.

\begin{tabular}{|c|c|c|c|c|c|}
\hline Genotype & Cigarette smoking $^{a}$ & Cases, n (\%) & Controls, n (\%) & $\mathrm{OR}(95 \% \mathrm{CI})^{\mathrm{b}}$ & P-value \\
\hline \multicolumn{6}{|l|}{ MnSOD } \\
\hline $\mathrm{Val} / \mathrm{Val}$ & Never & $150(33.6)$ & $109(51.9)$ & 1.00 & \\
\hline $\mathrm{Val} / \mathrm{Ala}+\mathrm{Ala} / \mathrm{Ala}$ & Never & $44(9.9)$ & $20(9.5)$ & $1.79(0.95-3.37)$ & 0.073 \\
\hline $\mathrm{Val} / \mathrm{Val}$ & Ever & $201(45.1)$ & $72(34.3)$ & 1.00 & \\
\hline Val/Ala+Ala/Ala & Ever & $51(11.4)$ & $9(4.3)$ & $2.23(1.02-4.88)$ & 0.045 \\
\hline P for interaction & & & & 0.552 & \\
\hline \multicolumn{6}{|l|}{ CAT } \\
\hline $\mathrm{C} / \mathrm{C}$ & Never & $174(39.0)$ & $122(58.1)$ & 1.00 & \\
\hline $\mathrm{C} / \mathrm{T}+\mathrm{T} / \mathrm{T}$ & Never & $20(4.5)$ & $7(3.3)$ & $1.80(0.71-4.60)$ & 0.217 \\
\hline $\mathrm{C} / \mathrm{C}$ & Ever & $228(51.1)$ & $75(35.7)$ & 1.00 & \\
\hline $\mathrm{C} / \mathrm{T}+\mathrm{T} / \mathrm{T}$ & Ever & $24(5.4)$ & $6(2.9)$ & $1.20(0.46-3.12)$ & 0.708 \\
\hline P for interaction & & & & 0.387 & \\
\hline \multicolumn{6}{|l|}{ GPx1 } \\
\hline Pro/Pro & Never & $177(39.7)$ & $116(55.2)$ & 1.00 & \\
\hline Pro/Leu & Never & $17(3.8)$ & $13(6.2)$ & $1.06(0.47-2.41)$ & 0.894 \\
\hline Pro/Pro & Ever & $228(51.1)$ & $75(35.7)$ & 1.00 & \\
\hline Pro/Leu & Ever & $24(5.4)$ & $6(2.9)$ & $1.23(0.47-3.21)$ & 0.674 \\
\hline P for interaction & & & & 0.773 & \\
\hline
\end{tabular}

a: "Never" is nonsmokers and "Ever" is the combination of current smokers and ex-smokers.

b: adjusted for age, sex, and alcohol drinking.

TABLE 5: Association between MnSOD genotypes and risk of coronary artery disease severity stratified by cigarette smoking status.

\begin{tabular}{|c|c|c|c|c|}
\hline & \multicolumn{4}{|c|}{ CAD severity } \\
\hline & 0 & 1 & 2 & 3 \\
\hline \multicolumn{5}{|c|}{ Never cigarette smoking } \\
\hline \multicolumn{5}{|l|}{ MnSOD } \\
\hline $\mathrm{Val} / \mathrm{Val}$ & 109 & 51 & 48 & 51 \\
\hline $\mathrm{Val} / \mathrm{Ala}+\mathrm{Ala} / \mathrm{Ala}$ & 20 & 20 & 14 & 10 \\
\hline $\mathrm{OR}(95 \% \mathrm{CI})^{\mathrm{a}}$ & 1.00 & $2.07(0.98-4.37)$ & $1.91(0.81-4.52)$ & $1.45(0.58-3.62)$ \\
\hline P-value & & 0.056 & 0.140 & 0.431 \\
\hline \multicolumn{5}{|c|}{ Ever cigarette smoking } \\
\hline \multicolumn{5}{|l|}{ MnSOD } \\
\hline Val/Val & 72 & 67 & 54 & 80 \\
\hline $\mathrm{Val} / \mathrm{Ala}+\mathrm{Ala} / \mathrm{Ala}$ & 9 & 20 & 13 & 18 \\
\hline $\mathrm{OR}(95 \% \mathrm{CI})^{\mathrm{a}}$ & 1.00 & $2.63(1.07-6.44)$ & $2.11(0.81-5.54)$ & $1.73(0.69-4.36)$ \\
\hline P-value & & 0.034 & 0.129 & 0.245 \\
\hline
\end{tabular}

with significantly increased CAD risk only in ever cigarette smokers with one vessel disease $(\mathrm{OR}=2.63,95 \% \mathrm{CI}=1.07$ 6.44). CAD risk differences are neither seen in never cigarette smoking individuals, nor in ever smokers with more severe CAD.

\section{Discussion}

Atherosclerosis is focal thickening plaques located in coronary artery intima. A growing body of evidence shows that oxidative stress directly induce inflammation cascade and accelerate oxidation of LDL-C, causing atherosclerotic plaque instability and finally worsening of CAD [20]. Oxidative stress results from imbalance between ROS production and antioxidants capacity. Antioxidants defend cells against damage from free radicals and ROS, maintaining redox homeostasis. MnSOD, GPxl, and CAT are all endogenous enzymatic antioxidants $[21,22]$. In the current study, we focus on whether genetic polymorphisms of MnSOD, GPx1, and $C A T$ affect vulnerability or severity of CAD in Taiwan. Our 
study demonstrated a significantly elevated CAD risk in subjects with $M n S O D$ Val/Ala+Ala/Ala genotype, compared to those carrying Val/Val genotype. Noticeably, among the eversmoker subgroup, CAD risk in $M n S O D$ Val/Ala+Ala/Ala genotype patients increased even further.

MnSOD is the major mitochondrial antioxidant enzyme [21]. Earlier animal studies found MnSOD knock-out Drosophila failed to survive [23]. MnSOD Val-9Ala is a well-known polymorphism. Substitution for Alanine with Valine causes a conformational change in the mitochondrial-targeting domain and damages the enzymes function [21, 24]. This polymorphism has been linked to several diseases, including breast cancer, lung cancer, prostate cancer, dyslipidemia, macular diseases, neuropathies, and Parkinson's disease [2527]. This implicates the importance of oxidative injury in the underlying pathophysiology of these disease processes.

The correlation between atherosclerosis and $M n S O D$ polymorphism was also observed. Kakko et al. collected a sample of 989 middle-aged hypertensive and control Caucasians subjects in Finland. They found that $M n S O D$ Val/Val carriers were associated with higher carotid intimal media thickness, which indicates more severe atherosclerosis [28]. Souiden et al. discovered an approximately twofold risk of CAD in Tunisian men harbouring the Val/Val genotype [22]. These results are inconsistent with our data. Among these studies, the Val allele brings about increased atherosclerotic risk, while our data demonstrated a nearly twofold risk for CAD in those harbouring Ala alleles. However, another pulmonary artery hypertension study in Chinese population found results similar to ours [29]. This inconsistency may be due to the influence of interracial $M n S O D$ Ala allele frequency difference. The higher frequency of Ala allele was found as $48.3 \%$ in Caucasians and $25.1 \%$ in Arabs, respectively $[22,28]$. It was a discrepancy compared to our Chinese population which was $7.83 \%$. Therefore, the association between $M n S O D$ genetic variants and CAD risk may be influenced by ethnicity.

As mentioned earlier, numerous clinical or animal studies disclosed correlations between $M n S O D$ polymorphism and diseases. However, when looking into details, the results were actually inconsistent and confusing. Whether the genetic variant brings about extra risk or benefit for disease varies across races, ethnics, and disease entities [30]. For instance, the Val allele has been shown to increase cardiovascular risks in most studies, but is probably protective to Parkinson's disease. Although seemingly incoherent with other study results, our study provides a scope of how MnSOD Val9Ala affects CAD in the local Taiwanese population and demonstrates a probable interaction with cigarette smoking.

GPxl is another intracellular antioxidant. Its deficiency is linked to atherogenesis [31]. GPxl activity is also independently associated with occurrence of CAD [32, 33]. GPx1 Pro198Leu is the best characterized polymorphism. Souiden et al. studied 164 patients with established CAD and 203 healthy controls and revealed a null association between GPXI polymorphism and CAD incidence and severity [22]. The discovery from Souiden et al. is in concert with our data. In contrast, Nemoto et al. reported that type 2 diabetes mellitus subjects carrying GPx1 Pro/Leu genotype have significantly higher calcium score than those with Pro/Pro genotype [34]. In Nemoto's study, the subjects were mainly type 2 diabetes patients. In comparison, only $11 \%$ in control group and $38 \%$ in CAD group have been diagnosed with DM in our population. Further studies are needed to clarify if there is association between DM and GPX1 polymorphism.

CAT is another major defense against oxidative stress [35]. Similarly, CAT polymorphism has been found to be correlated with several disease entities, including cancer, and metabolic diseases, etc. [36-38]. A meta-analysis analysed 10 case-control studies and concluded that CAT activity was associated inversely with CAD [39]. However, previous genetic analysis showed no differences in allele frequencies among diabetic patients with and without cardiovascular disease $[40,41]$. This is similar to our findings. However, the result may have been affected by difference of diabetes proportion since diabetes itself is an immense bias in genetically testing for CAD risk. The null association requires further confirmation.

The strength of our study is that all participants received coronary angiography to document CAD status. However, the subjects were enrolled from a single institute in Taiwan and probably represent only the local population. Besides, through angiography, we could only record the coronary vessel anatomy, but not physiological as fractional flow reserve. Therefore, latent CAD might be erroneously classified as healthy control. Current evidence about MnSOD, GPX1, and CAT polymorphisms are mostly observational, cross-sectional studies. To achieve better understanding on how antioxidants gene polymorphisms affect CAD, further well-conducted, large-scale, randomized control study is mandatory. In the meanwhile, the development of CAD is complex, requiring not only genetic predisposition, but also environmental factors. As shown in our study, cigarette smoking might interact with $M n S O D$ polymorphism, causing a higher risk in the ever-smoking subgroup. Future study may focus more on the interaction between MnSOD, GPx1, or CAT polymorphism and other environmental risk factors. The data of apoB-100 was not included which play an important role in the process of atherosclerosis. The interaction of apoB100 and genetic polymorphisms is not investigated in our study. Data from different races and ethnic groups should also be analysed independently.

\section{Conclusions}

Our results suggest $\mathrm{MnSOD} \mathrm{Val/Ala+Ala/Ala} \mathrm{genotypes} \mathrm{are}$ independent risk factors for susceptibility to CAD in the Taiwan population. There are no significant correlations between CAT (C-262T) and GPx1 (Pro198Leu) polymorphisms and CAD risk. Future large-scale, controlled trials are needed to further understand the interactions between these antioxidant gene polymorphisms and environmental factors. Local data in different ethnic groups are also important.

\section{Data Availability}

The data used to support the findings of this study were supplied by Dr. Chih-Ching Yeh under license and so cannot be 
made freely available. Requests for access to these data should be made to Dr. Chih-Ching Yeh by email: ccyeh@tmu.edu.tw.

\section{Disclosure}

An earlier version of this work was presented as an abstract at International Society for Environmental Epidemiology (ISEE) 2014.

\section{Conflicts of Interest}

The authors declare that there are no conflicts of interest regarding the publication of this paper.

\section{Acknowledgments}

This study was supported by Grants NSC 94-2314-B-039-031 from the National Science Council, Executive Yuan, Taiwan.

\section{References}

[1] E. J. Benjamin, M. J. Blaha, and S. E. Chiuve, "Heart Disease and Stroke Statistics-2017 Update: A Report From the American Heart Association," Circulation, vol. 135, 2017.

[2] G. A. Roth, M. D. Huffman, A. E. Moran et al., "Global and regional patterns in cardiovascular mortality from 1990 to 2013," Circulation, vol. 132, no. 17, pp. 1667-1678, 2015.

[3] R. B. D’Agostino Sr., R. S. Vasan, M. J. Pencina et al., "General cardiovascular risk profile for use in primary care: the Framingham heart study," Circulation, vol. 117, no. 6, pp. 743-753, 2008.

[4] P. Gæde, P. Vedel, N. Larsen, G. V. H. Jensen, H.-H. Parving, and O. Pedersen, "Multifactorial intervention and cardiovascular disease in patients with type 2 diabetes," The New England Journal of Medicine, vol. 348, no. 5, pp. 383-393, 2003.

[5] U. N. Khot, M. B. Khot, C. T. Bajzer et al., "Prevalence of conventional risk factors in patients with coronary heart disease," Journal of the American Medical Association, vol. 290, no. 7, pp. 898-904, 2003.

[6] L. G. Futterman and L. Lemberg, "Fifty percent of patients with coronary artery disease do not have any of the conventional risk factors," American Journal of Critical Care, vol. 7, no. 3, pp. 240244, 1998.

[7] E. S. Lander, L. M. Linton, B. Birren et al., "Initial sequencing and analysis of the human genome," Nature, vol. 409, no. 6822, pp. 860-921, 2001.

[8] The International HapMap Consortium, "A haplotype map of the human genome," Nature, vol. 437, pp. 1299-1320, 2005.

[9] S. Kathiresan and D. Srivastava, "Genetics of human cardiovascular disease," Cell, vol. 148, no. 6, pp. 1242-1257, 2012.

[10] H.-L. Yeh, L.-T. Kuo, F.-C. Sung, C.-W. Chiang, and C.-C. Yeh, "GSTM1, GSTT1, GSTP1, and GSTA1 genetic variants are not associated with coronary artery disease in Taiwan," Gene, vol. 523, no. 1, pp. 64-69, 2013.

[11] O. S. Kornfeld, S. Hwang, M. H. Disatnik, C. H. Chen, N. Qvit, and D. Mochly-Rosen, "Mitochondrial reactive oxygen species at the heart of the matter: new therapeutic approaches for cardiovascular diseases," Circulation Research, vol. 116, no. 11, pp. 1783-1799, 2015.

[12] C. Bănescu, A. P. Trifa, S. Voidăzan et al., "CAT, GPX1, MnSOD, GSTM1, GSTT1, and GSTP1 Genetic Polymorphisms in Chronic
Myeloid Leukemia: A Case-Control Study," Oxidative Medicine and Cellular Longevity, vol. 2014, Article ID 875861, 6 pages, 2014.

[13] C.-C. Yeh, F.-C. Sung, L.-T. Kuo, W.-P. Hsu, and H.-Y. Chu, "Polymorphisms of cytochrome P450 1A1, cigarette smoking and risk of coronary artery disease," Mutation Research Fundamental and Molecular Mechanisms of Mutagenesis, vol. 667, no. 1-2, pp. 77-81, 2009.

[14] P. J. Harris, V. S. Behar, M. J. Conley et al., "The prognostic significance of $50 \%$ coronary stenosis in medically treated patients with coronary artery disease," Circulation, vol. 62, no. 2, pp. 240-248, 1980.

[15] W. T. Friedewald, R. I. Levy, and D. S. Fredrickson, "Estimation of the concentration of low-density lipoprotein cholesterol in plasma, without use of the preparative ultracentrifuge," Clinical Chemistry, vol. 18, no. 6, pp. 499-502, 1972.

[16] J. E. Tanus -Santos, M. Desai, and D. A. Flockhart, "Effects of ethnicity on the distribution of clinically relevant endothelial nitric oxide variants," Pharmacogenetics, vol. 11, no. 8, pp. 719725, 2001.

[17] K. Hibi, T. Ishigami, K. Tamura et al., "Endothelial nitric oxide synthase gene polymorphism and acute myocardial infarction," Hypertension, vol. 32, no. 3, pp. 521-526, 1998.

[18] P. Lin, Y.-M. Hsueh, J.-L. Ko, Y.-F. Liang, K.-J. Tsai, and C.-Y. Chen, "Analysis of NQO1, GSTP1, and MnSOD genetic polymorphisms on lung cancer risk in Taiwan," Lung Cancer, vol. 40, no. 2, pp. 123-129, 2003.

[19] L. Forsberg, L. Lyrenäs, U. De Faire, and R. Morgenstern, "A common functional C-T substitution polymorphism in the promoter region of the human catalase gene influences transcription factor binding, reporter gene transcription and is correlated to blood catalase levels," Free Radical Biology \& Medicine, vol. 30, no. 5, pp. 500-505, 2001.

[20] T. Vichova and Z. Motovska, "Oxidative stress: Predictive marker for coronary artery disease," Exp Clin Cardiol, vol. 18, pp. e88-91, 2013.

[21] H. Fujimoto, J.-I. Taguchi, Y. Imai et al., "Manganese superoxide dismutase polymorphism affects the oxidized low-density lipoprotein-induced apoptosis of macrophages and coronary artery disease," European Heart Journal, vol. 29, no. 10, pp. 12671274, 2008.

[22] Y. Souiden, H. Mallouli, S. Meskhi et al., "MnSOD and GPx1 polymorphism relationship with coronary heart disease risk and severity," Biological Research, vol. 49, p. 22, 2016.

[23] A. Duttaroy, A. Paul, M. Kundu, and A. Belton, "A Sod2 Null Mutation Confers Severely Reduced Adult Life Span in Drosophila," Genetics, vol. 165, no. 4, pp. 2295-2299, 2003.

[24] M.-T. Moradi, K. Yari, Z. Rahimi, E. Kazemi, and M. Shahbazi, "Manganese superoxide dismutase (MnSOD Val-9Ala) gene polymorphism and susceptibility to gastric cancer," Asian Pacific Journal of Cancer Prevention, vol. 16, no. 2, pp. 485-488, 2015.

[25] A. Bag and N. Bag, "Target sequence polymorphism of human manganese superoxide dismutase gene and its association with cancer risk: a review," Cancer Epidemiology, Biomarkers \& Prevention, vol. 17, no. 12, pp. 3298-3305, 2008.

[26] H. Chen, M. Yu, M. Li et al., "Polymorphic variations in manganese superoxide dismutase (MnSOD), glutathione peroxidase-1 (GPX1), and catalase (CAT) contribute to elevated plasma triglyceride levels in Chinese patients with type 2 diabetes or diabetic cardiovascular disease," Molecular and Cellular Biochemistry, vol. 363, no. 1-2, pp. 85-91, 2012. 
[27] K. Pourvali, M. Abbasi, and A. Mottaghi, "Role of superoxide dismutase 2 gene Ala16Val polymorphism and total antioxidant capacity in diabetes and its complications," Avicenna Journal of Medical Biotechnology, vol. 8, no. 2, pp. 48-56, 2016.

[28] S. Kakko, M. Päivänsalo, P. Koistinen, Y. A. Kesäniemi, V. L. Kinnula, and M. J. Savolainen, "The signal sequence polymorphism of the MnSOD gene is associated with the degree of carotid atherosclerosis," Atherosclerosis, vol. 168, no. 1, pp. 147152, 2003.

[29] M. Xu, M. Xu, L. Han et al., "Role for Functional SOD2 Polymorphism in Pulmonary Arterial Hypertension in a Chinese Population," International Journal of Environmental Research and Public Health, vol. 14, no. 3, p. 266, 2017.

[30] A. Sutton, H. Khoury, C. Prip-Buus, C. Cepanec, D. Pessayre, and F. Degoul, "The Ala16Val genetic dimorphism modulates the import of human manganese superoxide dismutase into rat liver mitochondria," Pharmacogenetics, vol. 13, no. 3, pp. 145157, 2003.

[31] D. Wickremasinghe, H. Peiris, L. G. Chandrasena, V. Senaratne, and R. Perera, "Case control feasibility study assessing the association between severity of coronary artery disease with Glutathione Peroxidase-1 (GPX-1) and GPX-1 polymorphism (Pro198Leu)," BMC Cardiovascular Disorders, vol. 16, no. 1, article no. 111, 2016.

[32] S. Blankenberg, H. J. Rupprecht, C. Bickel et al., "Glutathione peroxidase 1 activity and cardiovascular events in patients with coronary artery disease," The New England Journal of Medicine, vol. 349, no. 17, pp. 1605-1613, 2003.

[33] R. Schnabel, K. J. Lackner, H. J. Rupprecht et al., "Glutathione peroxidase- 1 and homocysteine for cardiovascular risk prediction: results from the atherogene study," Journal of the American College of Cardiology, vol. 45, no. 10, pp. 1631-1637, 2005.

[34] M. Nemoto, R. Nishimura, T. Sasaki et al., "Genetic association of glutathione peroxidase-1 with coronary artery calcification in type 2 diabetes: a case control study with multi-slice computed tomography," Cardiovascular Diabetology, vol. 6, no. 1, article 23, 2007.

[35] E. Birben, U. M. Sahiner, C. Sackesen, S. Erzurum, and O. Kalayci, "Oxidative stress and antioxidant defense," World Allergy Organization Journal, vol. 5, no. 1, pp. 9-19, 2012.

[36] J. Kodydkova, L. Vavrova, M. Kocik, and A. Zak, "Human catalase, its polymorphisms, regulation and changes of its activity in different diseases," Folia Biol (Praha), vol. 60, pp. 153167, 2014.

[37] Y. Shen, D. Li, P. Tian et al., "The catalase C-262T gene polymorphism and cancer risk: a systematic review and meta-analysis," Medicine, vol. 94, no. 13, article e679, 2015.

[38] M. Hebert-Schuster, E. E. Fabre, and V. Nivet-Antoine, "Catalase polymorphisms and metabolic diseases," Current Opinion in Clinical Nutrition \& Metabolic Care, vol. 15, no. 4, pp. 397402, 2012.

[39] M.-I. Covas, G. Flores-Mateo, P. Carrillo-Santisteve et al., "Antioxidant enzyme activity and coronary heart disease: Metaanalyses of observational studies," American Journal of Epidemiology, vol. 170, no. 2, pp. 135-147, 2009.

[40] K. G. dos Santos, L. H. Canani, J. L. Gross, B. Tschiedel, K. E. P. Souto, and I. Roisenberg, "The Catalase -262C/T Promoter Polymorphism and Diabetic Complications in Caucasians with Type 2 Diabetes," Disease Markers, vol. 22, Article ID 983408, 5 pages, 2006.

[41] M. Flekac, J. Skrha, J. Hilgertova, Z. Lacinova, and M. Jarolimkova, "Gene polymorphisms of superoxide dismutases and catalase in diabetes mellitus," BMC Medical Genetics, vol. 9, article 30, 2008. 